cases, the dune pattern arises not by reflecting the pattern of the fluid flow itself, but rather is an inevitable result of the coupling between fluid flow and sand transport (a result mirroring conclusions from an earlier modelling effort to explain regularly spaced beach cusps $^{7}$ ).

What might we learn from this approach? Although the terminology of phase space and attractors may be foreign to the general geomorphological and geological community, we have a great deal to gain from casting these complex problems into this arena. Many of the problems faced by those interested in landscape evolution involve linked processes whose microphysics and chemistry tangle to create complicated landscapes. Werner's demonstration of the utility of this approach in the relatively simple case of sand dunes should inspire other geomorphologists to explore models of more complex landscapes for sets of simplifying rules.

Robert S. Anderson is in the Department of Earth Sciences, University of California Santa Cruz, Santa Cruz, California 95064, USA.

\title{
Getting oneself together
}

\section{Philip Ball}

"WHEN I use a word, it means just what I choose it to mean-neither more nor less." That pronouncement could surely come from the lips of materials scientists talking about self-organization and selfassembly (although its true originator, Humpty Dumpty, was no master of the latter). Substitute 'phase separation' or 'crystallization' and at a stroke your work sounds decades older. Nonetheless, striking examples of the spontaneous ordering and patterning of materials can be found that genuinely warrant such neologisms; and several were described at a recent meeting of the Materials Research Society*.

At the meeting, an entire symposium was devoted to self-assembled materials, which found itself both figuratively and literally next door to that on materials inspired by biology. Yet it was elsewhere that Jerry Tersoff (IBM) was to be found explaining how deceptively simple physics can be an organizational force too. Picture crystals of a germanium-silicon alloy growing on the surface of silicon. The surface energies are such that the crystals grow as tiny, faceted pyramids, the corners of a cubic phase. These are self-assembled quantum dots in some people's books, humble semiconductor crystallites in others. Their growth follows random heterogeneous nucleation events, so that the silicon surface becomes sprinkled with pyramids of varying size. All this is possibly useful for quantum device construction, but it is hard to control and is scarcely unexpected.

Now bury these pyramids under another layer of silicon, so that the tips lie under several atomic layers. Then start to deposit the $\mathrm{Ge}-\mathrm{Si}$ alloy again. The first surprise is that the new crystallites grow directly over the tips of those buried below. The second is that this is an inexact description only insofar as the new array of quantum dots

* Fall Meeting of the Materials Research Society, Boston, USA, 27 November - 1 December 1995. looks a little more orderly - there is a correlation between pyramids in the first and second layers, but also a lateral correlation between those within the second layer. Repeat this sequence of burial and new pyramid growth, and the new array is even more correlated - the pyramids look reasonably well aligned into rows, and of more uniform size. For those who do not trust the eye, a Fourier analysis of microscopic images shows a progression from a blurred ring to symmetric, sharpening spots.

These experiments, not yet published, were done by researchers at the University of Wisconsin and at AT\&T Bell Laboratories. They set Tersoff, a theorist, thinking about the origin of the self-organization. The correlations between crystallite positions in successive layers are easily understood: they are a consequence of the strain imposed on the atomic planes of the silicon overlayer by the embedded Ge-Si crystals, which is maximal over their tips and decays to all sides. This strain, transmitted to the uppermost silicon layer, creates a higher surface energy and thereby enhances the possibility of crystallite nucleation.

But what of the gradual ordering and size uniformity? Tersoff first developed a two-dimensional model of the process, although later confirming that it works for three dimensions too. His calculations show that, if two buried pyramids are close enough together, their strain maxima coalesce into a single peak. That is not astonishing, but it is enough almost by itself to generate increasing order in successive layers of crystallites. The only other factor needed is that between widely spaced buried pyramids there is a very shallow strain maximum more or less equidistant from the two, so a 'fresh' crystallite is most likely to nucleate halfway between them. In this way, large gaps in an underlying layer are filled in the succeeding one.

Tersoff shows that these two factors act as a kind of band-pass filter for crystallite nucleation, preventing two from forming too close together (as the strain maxima from below coalesce) or staying too far apart (as new crystallites will fill in the gaps). Very quickly (after about six iterations), an initially random distribution of pyramids turns into a fairly regular array. But the distribution never becomes fully ordered, because the band-pass filter gives diminishing returns, always allowing some noise to remain in the pass band.

If perfectly ordered quantum dots are what you need, Moungi Bawendi and colleagues at the Massachusetts Institute of Technology have a better solution, as related by Chris Murray from Bawendi's group. $\mathrm{He}$ is to be commended for resisting the temptation to pass off as 'self-organization' a process that is in fact colloidal crystallization - but the degree of organization here is nevertheless phenomenal. Bawendi's group have synthesized nanometre-scale clusters of crystalline semiconducting cadmium selenide of essentially uniform size and spherical shape, whose size dispersion is less than an atomic layer (Science 270, $1335-1338 ; 1995)$. They prepare the crystals under conditions in which nucleation and growth are closely controlled to give particle sizes within the range of 2 to 15 nanometres, and then passivate the surfaces by attaching organic substituents trialkylphosphines, which give each cluster a hairy coat of hydrophobic alkyl tails. These 'soft' balls will coalesce from dispersions in polar alcohols, because of colloidal interactions, forming perfectly regular, hexagonally packed superlattices colloidal crystals that are large and robust enough to be picked up with tweezers.

Colloidal crystallization is well known for polymer spheres of micrometre size, dispersions of which settle into iridescent crystals which are loosely bound and easily disrupted by shear. And aggregation of silica spheres in the natural environment leads to opal, with its pearly lustre. But the MIT group have done far more than simply shrink to the nanoscale conventional stuff from the microscale. Cadmium selenide clusters this small have their electronic band structure modified by quantum size effects - the well-known particle-in-a-box idea - bringing their bandgap into the visible part of the spectrum and making it continuously tunable as a function of size. So these materials are potentially electroluminescent media for light-emitting devices; indeed, CdSe clusters have been used in such (see V. L. Colvin et al. Nature 370, 354; 1994). The control that Bawendi and colleagues have over size (and thus absorption and emission wavelength) and crystallization properties (the superlattice spacing can be varied by altering the alkyl chain length, for example) promises to make these nanocluster arrays a serious contender in the burgeoning field of display devices.

Philip Ball is an associate editor of Nature. 